Andrews, George D, COMOSU '86; 3608 Pilgrim Ln, Altus, OK 73521-1000

Auxter, Thomas Matthew, KCOM '86; 801 New York, Humboldt, KS 66748-1801

Benton, Regis A, UOMHS '53; 1187 W Bristol, Flint, MI 48507-5517

Berry, Jolene R, WVSOM '86; Davis Stuart Rd, Route 2 Box 171, Lewisburg, WV 24901

Carothers, Allen H, KCOM '71; No 302, 715 W Michigan, Jackson, MI 49201-1915

Cooper, William G, UHSCOM '73; Box 37, Saltillo, TN 38370-0037

Curry, J Michael, TCOM '87; 1276 Hurst Dr, Enid, OK 73703

Geiger, A John, UHSCOM '77; PO Box 99, Wynnewood, OK 73098-0099

Ghafouri, Mohammad, MSUCOM '90; No 12, $1450 \mathrm{E}$ Pond St, Okemos, MI 48864

Halley, James A Cdr, WVSOM 78; 714 Balmoral Ln, Orange Park, FL 32073-4287

Harrison, William J, COMOSU ' $81 ; 16$ th and University, PO Box 1610, Durant, OK 74702

Hood, Louie Franklin, UHSCOM '83; 512 Chestnut St, Grenada, MS 38901-5539

Ilowite, Norman H, PCOM '55; 1563 Center Ave, Ft Lee, NJ 07024-4602

Kaplan, Henry B, TCOM '86; Branch Clinic, NAS Chase Field, Beeville, TX 78103

Keith, Rosalie C, UNECOM '85; Box 197, 2nd General Hospital, APO NY, NY 09180

Kravanya, Margaret A, KCOM '69; 24300 Chagrin Blvd, Beachwood, OH 44122-5629 Landau, Les Lt Cdr, UHSCOM '84; US Naval Hospital, PO
Box 2907, FPO SEA, WA 98778-5000

Lapp, Richard L, UHSCOM '83; 5806 Lake Underhill Rd, Orlando, FL 32807

Madsen, Kurt R, KCOM '88; 916 E Line, Kirksville, MO 63501

Mendelson, Sol, COMP ' $82 ; 6790$ Hellman, Alta Loma, CA 91701-4828

Miller, Gregory L, OUCOM '84; No. I3, $2801 \mathrm{~S}$ Main St, Findlay, $\mathrm{OH}$ 45840-1063

Novak, Glenn J, UOMHS '79; 51 Westchester Dr, Youngstown, OH 44515-3900

O'Gorman, Joseph D, UHSCOM '86; 119 Waters Edge Dr, Ocean Springs, MS 395645118

Onderko, Gregory J, UOMHS '86; No 512, 11020 Bristol Bay Dr, Bradenton, FL 34209-7910

Orbeck, Kenneth P, UOMHS '87; 1000 Harrington Blvd, Mount Clemens, MI 48043-2992

Owens, Bruce, UHSCOM '87; PO Box 197, Channahon, IL 60410

Perez, Michael, UOMHS '88; No 408, 291 Scarborough, Conroe, TX 77304

Schreibman, Walton M, PCOM '53; 1007 S Main St, Pleasantville, NJ 08232-3619

Silverman, Russell V, PCOM '70; 7355 Malvern Ave, Philadelphia, PA 19151-2210

Silverstein, Arthur, CCOM '73; 691 Morro Ave, Morro Bay, CA 93442-2298

Silverton, Kimball, KCOM '85; 1246 Dyemeadow, Flint, MI 48532-2318

Walter, Margaret Harris, TCOM '87; No 104, 2000 Esters Rd, Irving, TX 75061

Waxman, Frank M, PCOM '72; 9800 W Bay Harbor Dr, Bay Harbor, FL 33154

\section{SURGICAL RESIDENTS/FELLOWS}

Clinical residency/fellowship positions available in adult and pediatric cardiothoracic surgery.

- Large volume of cases -New Jersey license required -Excellent salary and benefits

Please contact:

Lynn B. McGrath, MD Chairman, Dept. of Surgery (609) 893-6611, Ext. 359

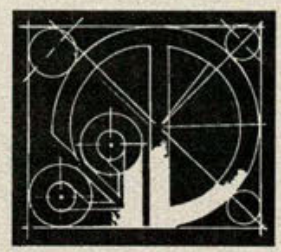

Deborah Heart and Lung Center 200 Trenton Road Browns Mills, NJ 08015

\section{FELLOWSHIP IN VASCULAR SURGERY}

The Deborah Heart and Lung Center is currently seeking qualified applicants for a Vascular Fellow position. Please contact:

Lynn B. McGrath, MD Chairman, Dept. of Surgery (609) 893-6611, Ext. 359

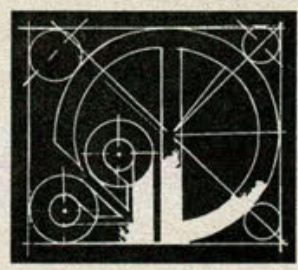

\section{Deborah Heart and Lung Center 200 Trenton Road Browns Mills, NJ 08015}

